\title{
Kinematics and End-Point Control of Arm Movements Are Modified by Unexpected Changes in Viscous Loading
}

\author{
Jerome N. Sanes \\ Laboratory of Neurophysiology, National Institute of Mental Health, and Human Motor Control Section, Medical \\ Neurology Branch, National Institute of Neurological and Communicative Disorders and Stroke, Bethesda, \\ Maryland 20892
}

\begin{abstract}
These experiments were undertaken to evaluate whether the kinematics and end-point control of learned movements were affected by changes in dynamic loads or were determined largely by centrally specified motor programs. Human subjects performed flexion movements about the wrist in a discrete visual tracking task for a range of movement sizes. For some movements, viscosity was increased at movement onset. When the viscous load opposed movement unexpectedly, subjects initially overshot the intended target for all movement sizes, but only for the smaller movements did the overshoot persist. Unexpected introduction of heavier loads was more effective in inducing these behavioral changes; the lightest loads did not alter end-point positioning. When subjects had visual guidance about performance when load changes occurred, the effect of the unexpected occurrences of viscous loads was diminished, suggesting that subjects rapidly adjusted their movement strategy, depending on task demands and performance. The movement responses were mediated by short-latency and long-duration muscle responses triggered by the change in viscous loading. Although the triggered muscle responses were larger when the loads were encountered during performance of large, in comparison to small, movements, smaller muscle responses affected small movements more than large triggered responses did large movements. This suggests that triggered muscle responses are compensatory in certain movement situations but disruptive in others. In addition, these findings demonstrated that dynamic loads especially affect the kinematics and end-point control of smaller movements, suggesting that kinesthetic inputs and central motor commands interact so subjects may achieve accurate positioning for certain classes of movements.
\end{abstract}

Several recent studies of motor control in patients with sensory loss have described impairments of movement accuracy as a result of viscous-load changes occurring during movement (Day and Marsden, 1982; Nam et al., 1984; Rothwell et al., 1982a; Sanes et al., 1985). The reason for the use of viscous loads (as compared to springs or weights) in these studies was that viscous loads acted during displacement but not during absence of movement. Thus, if a subject is to displace the wrist from one angle to another at a fixed angular velocity, an unexpected change of viscosity will require changed muscular output during the displacement between the starting position and the terminal

\footnotetext{
Received Dec. 12, 1985; revised Apr. 14, 1986; accepted May 30, 1986.

The author expresses his deepest gratitude to Dr. Edward V. Evarts for his tireless and selfless encouragement and suggestions throughout all aspects of this work. His impact on this and similar studies was profound and will be missed sorely in the future. Lori Budzinski is thanked for assistance in preparing the illustrations.

Correspondence should be addressed to Dr. Jerome N. Sanes, Human Motor Control Section, NINCDS, Building 10, Room 5N-226, Bethesda, MD 20892. $0270-6474 / 86 / 113120-08 \$ 02.00 / 0$
}

position, but will not require any change in muscular activity during maintenance of the initial or terminal positions. It has at times been proposed (Kelso and Holt, 1980; Lestienne et al., 1981; Polit and Bizzi, 1979; but see Hasan and Enoka, 1985; Matheson et al., 1985) that CNS outputs can specify steadystate limb positions in the absence of kinesthetic feedback. According to this notion, an increase of viscosity opposing the movements of both normal and deafferented subjects would alter the intended velocity during movement from the initial to the terminal position, but would have no effect on intended terminal accuracy. These considerations underlie the theoretical implications of recent findings that deafferented humans fail to position a limb accuratcly after a viscous load is introduced during movement (Day and Marsden, 1982; Rothwell et al., 1982a; Sanes, 1983; Sanes et al., 1985). As mentioned above, if CNS signals in the absence of somesthetic feedback were able to specify movement end-points, then a change of viscous loading would alter movement trajectory but would not affect movement end-point. However, Day and Marsden (1982) demonstrated that digital anesthesia (which eliminated the reflex muscle responses that normally occurred when subjects encountered an unexpected resistance) caused a failure to compensate for the effects of the viscous load. The movements evaluated by Day and Marsden (1982) were relatively large. Since somesthetic afferents have their greatest sensitivity for small-amplitude signals (e.g., Hasan and Houk, 1975; Knibestöl and Vallbo, 1980; Matthews and Stein, 1969), and small movements are more affected by peripheral perturbations than are large movements (Sanes and Evarts, 1983a, b), it seemed likely that the effects of viscous-load changes would vary in relation to movement size. The present study in normal subjects was designed to pursue these issues by determining the effects of unpredictable changes of viscosity during movements of different sizes. A subsidiary concern was to investigate whether subjects could adjust their motor responses to unexpected perturbations when knowledge about movement errors was provided.

\section{Materials and Methods}

Ten healthy, right-handed subjects ( 6 females, 4 males) between the ages of 18 and 33 years were studied. All subjects were naive with respect to the design and goals of the experiment. The experimental arrangement is illustrated in Figure $1 A$. The right forearm was stabilized and the right hand was placed between two lightly padded metal plates that were coupled to a low-friction, brushless, DC torque motor (Aeroflex TQ64). The hand was hidden from view and positioned to allow flexion-extension movements of the wrist, with the wrist joint positioned directly over the axle of the torque motor. Subjects viewed 3 vertically oriented lines on a large-display Wavetek oscilloscope (Model 1901B). Two spatially separated lines represented a target window (target cursor) and the third line corresponded to the orientation of the torque motor handle and subject's hand (position cursor). Subjects were instructed to orient the handle such that the position cursor was within the target cursor 
A.

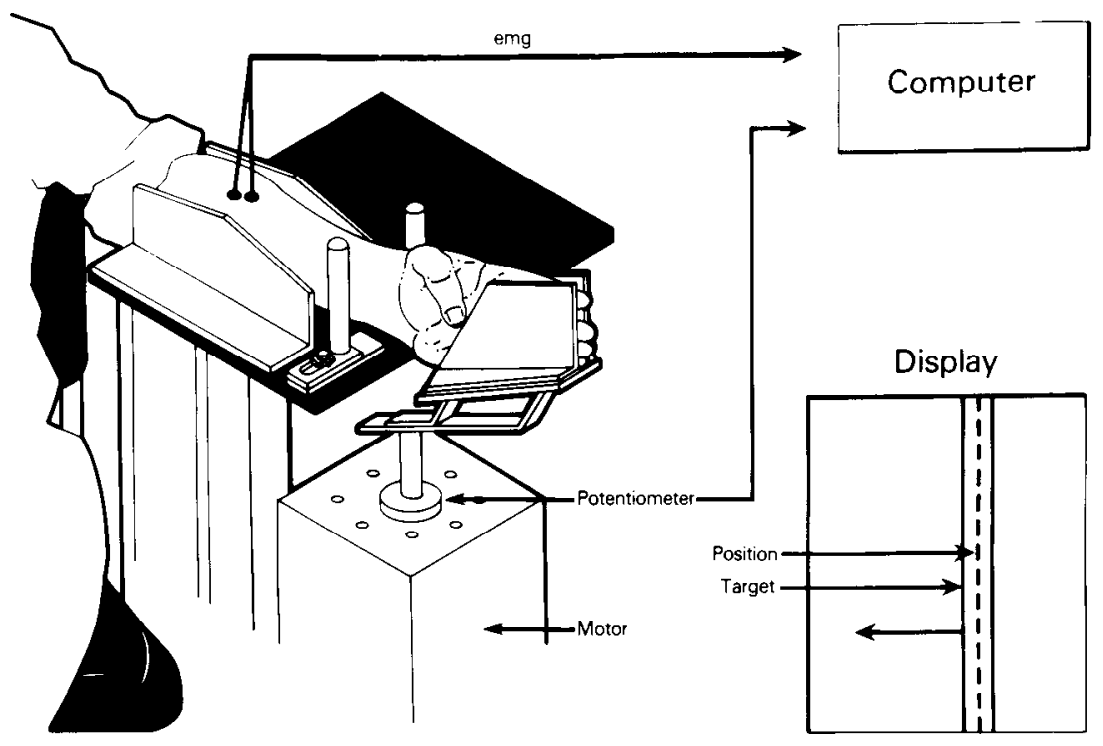

B.

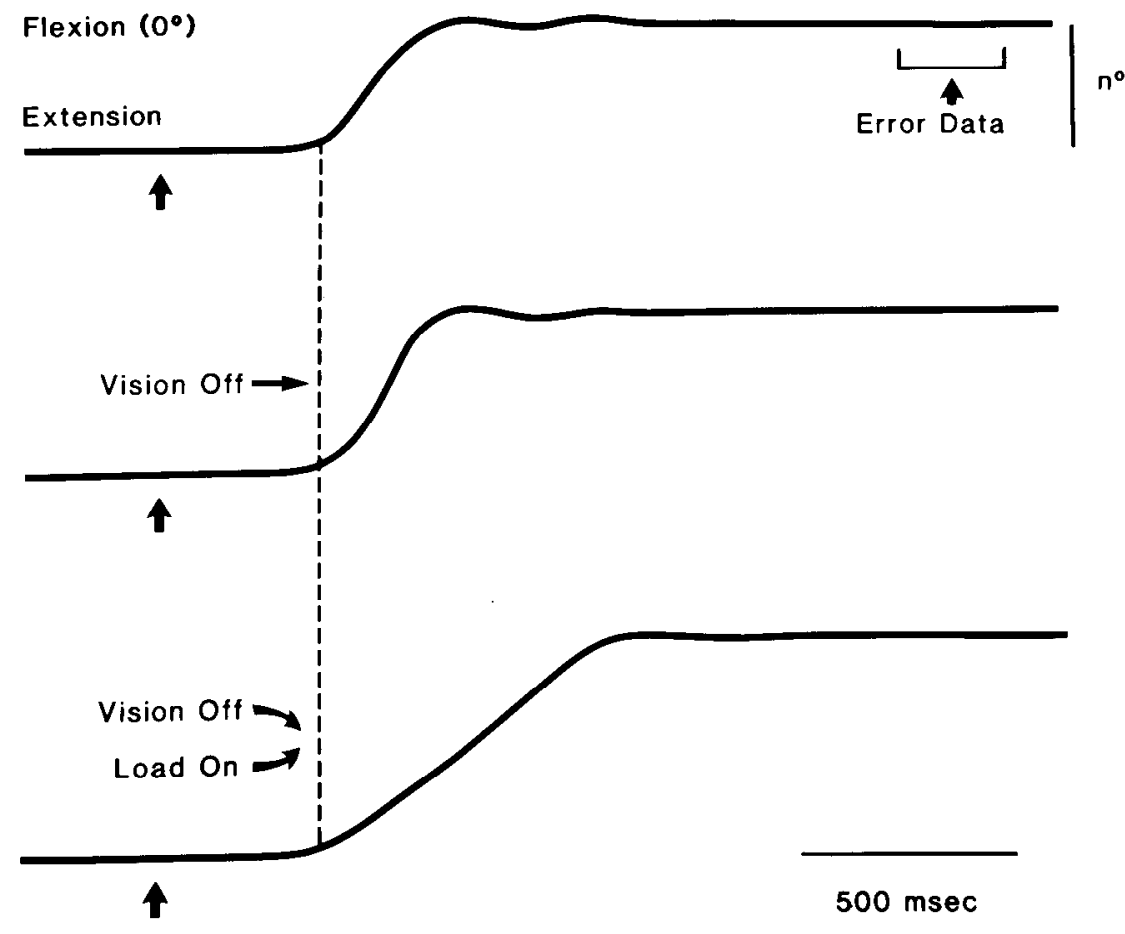

Target Jump

Figure 1. Schematic of experimental apparatus and data analysis techniques. Traces in $B$ represent hand position; 1 line for each of the 3 trial types in the major experiment. The dashed line indicates that the load or visual guidance (or both) was changed at movement onset. Dynamic error was mcasurcd at peak position (zerocross velocity), while end-point error was measured $1-1.3 \mathrm{sec}$ after this point.

and to reestablish alignment between position and target cursors when the target cursor jumped to a new location. The magnitude of handle rotation that was necessary to establish realignment after the target jumped was $30^{\circ}$ in certain blocks of trials, $10^{\circ}$ in other blocks, and $3^{\circ}$ in still other blocks. The distance on the video screen between the hold zone and the final target was constant, but the amount of angular displacement necessary to realign the position and target cursors varied according to movement size. For all trials, the target cursor remained displaced from the center of the display until subjects moved the handle to reestablish and then maintain alignment for $1.5-2.5 \mathrm{sec}$ in a small zone ( $\pm 5 \%$ of the movement size) around the midpoint of the target window. The target cursor was located such that wrist extension $\left(3^{\circ}\right.$, $10^{\circ}$, or $30^{\circ}$ ) was required to initially align the position and target cursors. The hold period was restarted if a subject failed to hold the handle within the target window for the entire hold period. At the end of this period, the target cursor jumped to the middle of the video display. Subjects were instructed to move the handle as quickly and as accurately as possible to match the position and target cursors. The final position for all movements was $0^{\circ}$ of wrist rotation.

Subjects participated in a single practice session and then several test 
A

Figure 2. End-point error in degrees $(A)$ and in proportion to movement size $(B)$, in relation to movement size for the group of subjects. Data are shown only for the experiment in which the heaviest load unexpectedly opposed movement. The error bars are 1 SEM and, if missing, indicate that the variability was too low to display. The plus and minus symbols in this and Figure 7 indicate whether the load or visual guidance was on (plus) or off (minus) during movement.

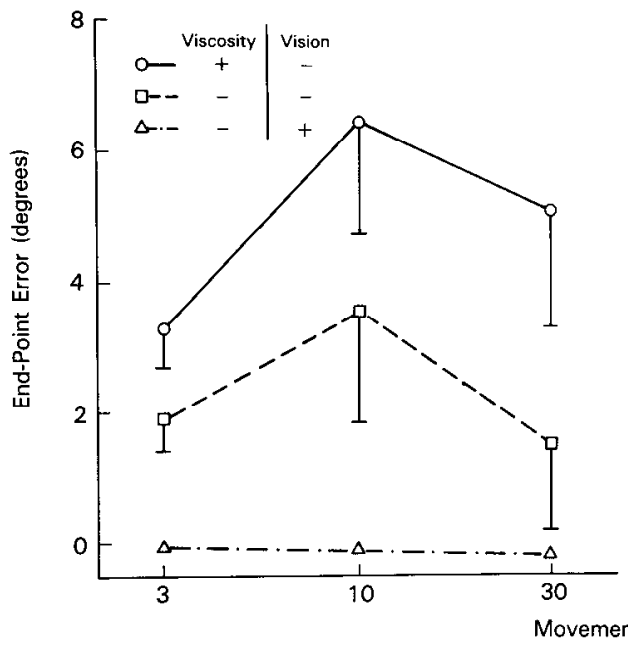

B

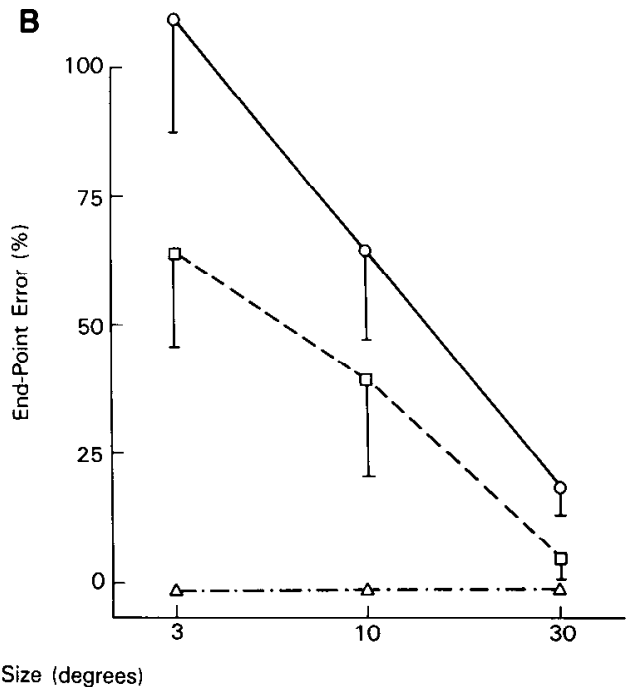

sessions. One or 2 test procedures were completed in each visit. Across sessions, the blocks of each movement size were performed in a counterbalanced order. In the practice session, and separately for each movement amplitude, subjects performed 250 wrist flexion movements when the position and target cursors were available continuously to guide limb movement and end-point positioning. Improvements in accuracy and increases in movement speed were encouraged until the initial phases of most movements $(>70 \%)$ were performed within $250 \mathrm{msec}$, with an initial accuracy of $\pm 15 \%$. Subjects had no experience moving with viscous loads or without visual guidance during the practice session.

In the test procedures, 100 trials were performed and 2 variables were manipulated: (1) the availability of visual guidance and (2) the unexpected introduction of a viscous load when movement began. Figure $1 B$ illustrates how visual guidance and the change in viscous loading occurred. On one-half of the trials, the position cursor remained visible throughout the movements and final positioning and there was no change in the viscous loading. For the remaining one-half of the trials, the position cursor disappeared when a velocity threshold was crossed. This threshold was set such that the visual guidance was removed almost as soon as movement began. On one-half of the trials without visual guidance, an increase in viscous loading occurred simultaneously with the disappearance of the position cursor. In each of 3 experiments, the

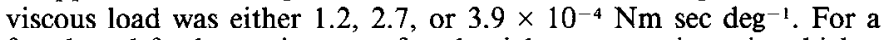
fourth and final experiment, a fourth trial type was given, in which a viscous load of $3.9 \times 10^{-4} \mathrm{Nm} \mathrm{sec} \mathrm{deg}^{-1}$ was introduced at movement onset while visual guidance remained available. In this experiment, all trial types occurred with an equal probability of 0.25 .

For the test procedures, additional instructions were given to augment those provided for practice. The new instructions stipulated that when visual guidance was removed, movements should be made in a manner similar to visually guided movements (that is, rapidly and accurately) and that the hand should be made stable as soon as possible. Subjects were discouraged from attempting "blind" corrections without the availability of visual guidance. For trials with changes in viscous loading, subjects were instructed to make similar movements, but to compensate, as best as possible, for the changes in viscosity. Trials were presented in an unpredictable order and no cues were available to indicate the type of upcoming trial. At the end of individual trials without visual guidance, the hand was passively moved with a smooth servo-controlled ramp from the end-point position on that trial to the initial position for all trials. The passive movement lasted for $1 \mathrm{sec}$ and visual guidance was restored only when the hand reached the initial position. When subjects changed to a new movement size, practice trials were allowed ad libitum. Subjects needed between 5 and 50 practice trials to adjust to the new movement size. Surface electromyograms (EMG) were recorded from the wrist flexor and extensor muscles using conventional methods. The signals were amplified with Grass AC-coupled high-inputimpedance amplifiers ( $30 \mathrm{~Hz}$ low- and $3 \mathrm{kHz}$ high-filter cutoffs) and stored on direct channels of an analog tape recorder. EMGs were subsequently rectified and low-pass-filtered (Gottlieb and Agarwal, 1970) before digitization at $100 \mathrm{~Hz}$ with a PDP $11 / 34$ computer. The hand position and velocity signals and an event signal were also tape recorded for off-line digitization.

Figure $1 B$ illustrates the general scheme for data analysis. Movement errors were measured when velocity first reached zero (dynamic error) and from 1000 to $1300 \mathrm{msec}$ (digitizing rate of $100 \mathrm{~Hz}$ ) after this point (end-point error). Errors in performance were expressed in degrees or in proportion to movement size. Error in degrees was the angular difference between the actual handle position and the handle position called for by the target cursor, whereas proportionate error was expressed in relation to each movement size. Movement time (MT) was measured to within $10 \mathrm{msec}$ accuracy from the first change in velocity after the target cursor jumped to the middle of the display to the first zero-cross in velocity. Dynamic error, end-point error, and MT were averaged across conditions and analyzed with repeated-measures analyses of variance (Statistical Analysis System, North Carolina) and with pairedcomparison procedures.

\section{Results}

\section{End-point error}

The major features of the effects of the unexpected introduction of the heavicst viscous load for end-point crror, expressed in degrees, are displayed in Figure $2 A$. Subjects overshot the intended target when movements were performed without visual guidance $(p \leq 0.01)$ and the introduction of a viscous load further increased end-point error (paired $t$ tests; $p \leq 0.02$ ). Although it appears that end-point error was greatest for the $10^{\circ}$ movement, the analysis of performance with all 3 loads failed to show a significant effect of movement size on end-point error. If the experiment in which subjects encountered changes with only the heaviest viscous load is considered by itself, there was a main effect of movement size on end-point error $(p \leq 0.025)$. Increases in the viscous load resulted in greater overshot $(p \leq$ $0.0001)$.

The end-point error data, expressed in proportion to movement size, are illustrated in Figure $2 B$. Removal of visual guidance at movement onset resulted in overshoot regardless of the initial loading conditions ( $p \leq 0.005$ ). In addition, proportionate end-point error increased during the performance of smaller movements $(p \leq 0.0001)$. The interaction between movement size and the presence or absence of a viscous load at movement onset was not significant. However, paired comparisons showed that end-point error was greater when any of the viscous loads unexpectedly opposed movement than with unopposed control movements $(p \leq 0.02)$. The difference between end-point error for nonvisually guided test trials (that is, load change) and for 


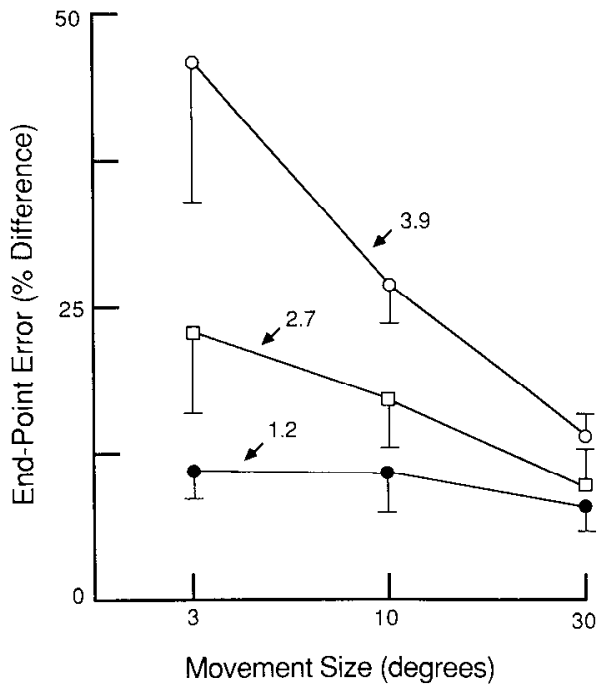

Figure 3. Proportionate difference error in relation to movement size. Difference in end-point error between nonvisually guided trials in which 1 set of trials was performed without introduction of a viscous load and the second set of trials was performed with the unexpected introduction of the load at movement onset. Results for all 3 loads (numbers next to arrows are in units of $10^{-4} \mathrm{Nm} \mathrm{sec} \mathrm{deg-1)} \mathrm{are} \mathrm{shown} \mathrm{for} \mathrm{the} \mathrm{group} \mathrm{of}$ subjects. The medium and heavy load causcd a grcatcr proportionatc end-point overshoot for the smaller movements. The lightest load had little effect on end-point error, suggesting a threshold effect for the unexpected introduction of viscous loads.

nonvisually guided trials for which the loading conditions were unchanged is shown in Figure 3 . Note that the amount of error was larger for small than for large movements $(p \leq 0.0025)$ and for heavier loads than for the change to lighter loads ( $p \leq 0.0005$ ).

One possible source of the increases in end-point error when visual guidance was withdrawn at the onset of movement was that subjects were never explicitly informed about their performance (that is, had no "knowledge of results"). This was the case for trials in which the loading conditions changed or did not change. The contribution of this lack of knowledge of results was evaluated when allowing permutation of visual guidance and load conditions. Thus, in addition to the trial types discussed above, a visually guided load increase trial was introduced. The results from this experiment are displayed in Figure 4 ; in general, the effects of visual guidance and movement size were roughly comparable to those observed in the previous experiments; movement error increased when visual guidance was removed $(p \leq 0.025)$ and when movement size decreased ( $p \leq 0.0001$ ). However, changing the loading conditions at the onset of movement had no significant effect upon end-point error.

\section{Muscle responses}

The average muscle activities from 1 subject, observed on trials with and without the viscous load, are shown in Figure 5. Since none of the movements were at or near maximum speed, the triphasic EMG pattern of reciprocal bursts in agonist and antagonist muscles was not usually ohserved; instead, a single burst in these muscles was characteristic. The agonist and antagonist bursts were graded according to movement size. When the viscous load was introduced, short-latency (35-60 msec) triggered muscle responses occurred in the agonist, and a commensurately short-latency silence occurred in the antagonist. The muscle responses to load change were graded in latency and amplitude such that earlier and larger, but not more prolonged, changes occurred when larger movements were obstucted by the unexpected increase in viscous loading. For all movement sizes, the

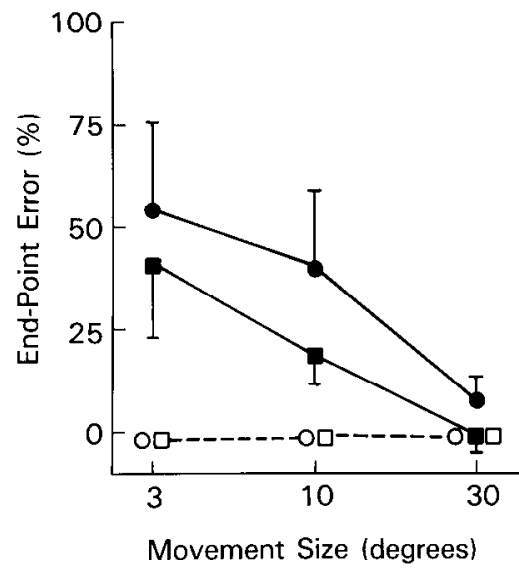

Figure 4. Proportionate end-point error when loaded trials were both visually and nonvisually guided. Filled symbols represent trials in which there was no visual guidance, open symbols those with continuous visual guidance. Circles represent trials in which the viscous load was introduced at movement onset, and squares indicate end-point error for trials in which the loading was unchanged.

agonist muscle was recruited more with the unexpected load increase, but the antagonist was silenced only during performance of the $30^{\circ}$ movements, moderately suppressed during the $10^{\circ}$ movements, and affected little when loads were introduced during performance of $3^{\circ}$ movements.

\section{Kinematics}

The MT data for all conditions are shown in Figure 6. Not surprisingly, the major determinant for MT was the presence or absence of a viscous load during movement. Introduction of viscosity at movement onset increased MT $(p \leq 0.0001)$. MT increased more when the heavier loads were introduced ( $p \leq$ 0.0001 ) and the time to complete smaller movements was affected more by increases in viscosity than by completion of larger movements $(p \leq 0.005)$.

The dynamic bias error data for the experiments when the heaviest viscous load was used are illustrated in Figure 7 . The analyses of dynamic error (in degrees) for all viscous loads will be presented first. The dynamic error increased when subjects encountered larger loads $(p \leq 0.01)$ at movement onset. Movement size influenced dynamic error such that the largest errors were observed for $10^{\circ}$ movements when the load was introduced $(p \leq 0.01)$. Bias errors for $3^{\circ}$ and $30^{\circ}$ movements did not differ from each other. The bias error was quite similar for all movement sizes when the lighter 2 loads were introduced unexpectedly, whereas (as noted above) dynamic error for the $10^{\circ}$ movements was larger than for $3^{\circ}$ and $30^{\circ}$ movements when the heaviest viscosity occurred at movement onset $(p \leq 0.05$; size $\times$ load interaction).

The results for the analysis of proportionate dynamic error were somewhat different than that for error expressed in degrees. First, the main effect of trial type (that is, viscous or no load) was not significant. Nevertheless, there was a significant interaction between movement size and presence or absence of load $(p \leq 0.05)$. Similar to the dynamic error data expressed in degrees, increases in viscosity increased percentage error ( $p \leq$ $0.001)$. There was an interaction between load magnitude and movement size such that, for the smaller movements, there was increasingly larger proportionate dynamic error when the loads increased $(p \leq 0.025)$.

\section{Discussion}

The major findings of the present experiments are consistent with previous results demonstrating that mechanical pertur- 
$3^{\circ}$

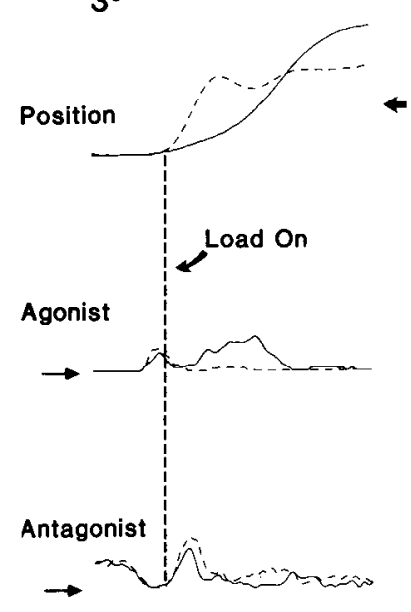

$10^{\circ}$

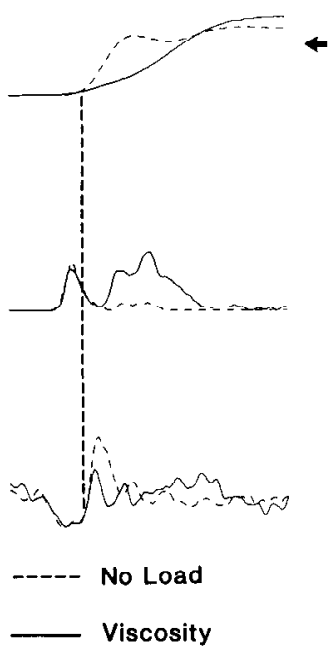

$30^{\circ}$

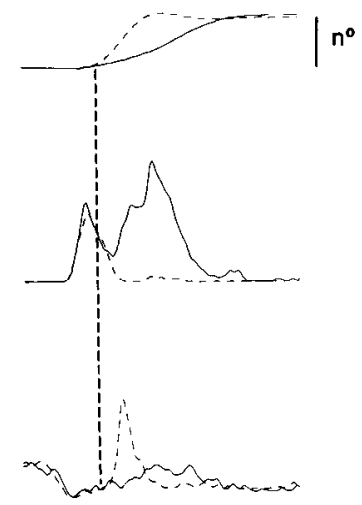

$200 \mathrm{~ms}$
Figure 5. EMG responses to changes in viscosity. Average hand position and EMGs for loaded (solid lines) and unloaded (dashed lines) trials, both performed without visual guidance, are superimposed. Introduction of the load-triggered long-duration muscle responses, which caused overshoot more for the smaller than for the larger movements. The arrows next to the $E M G s$ (left traces only) signify the baseline level of muscle activity. bations encountered during movement disrupt the performance of small movements (Sanes and Evarts, 1983a, b). The current results also support the conclusion reached from motor psychophysical evaluations of acutely or pathologically deafferented humans (Day and Marsden, 1982; Rothwell et al., 1982a; Sanes et al., 1985), that is, impaired proprioceptive sensation disrupts behavioral compensation for unexpected changes in dynamic loads occurring during movement. These results appear to conflict with previous experiments on surgically deafferented monkeys (e.g., Polit and Bizzi, 1979) and acutely deafferented humans (Kelso and Holt, 1980) which showed unimpaired movement accuracy following the introduction of unexpected mechanical perturbations during voluntary movement. However, as noted previously, the size and intended precision of movements are critical variables in predicting whether a perturbation occurring during movement will have adverse effects on movement kinematics and accuracy (Goodwin et al., 1972; Laszlo and Bairstow, 1971; Sanes and Evarts, 1983a, b). In addition, the tcchniques uscd by Kelso and Holt (1980) re-

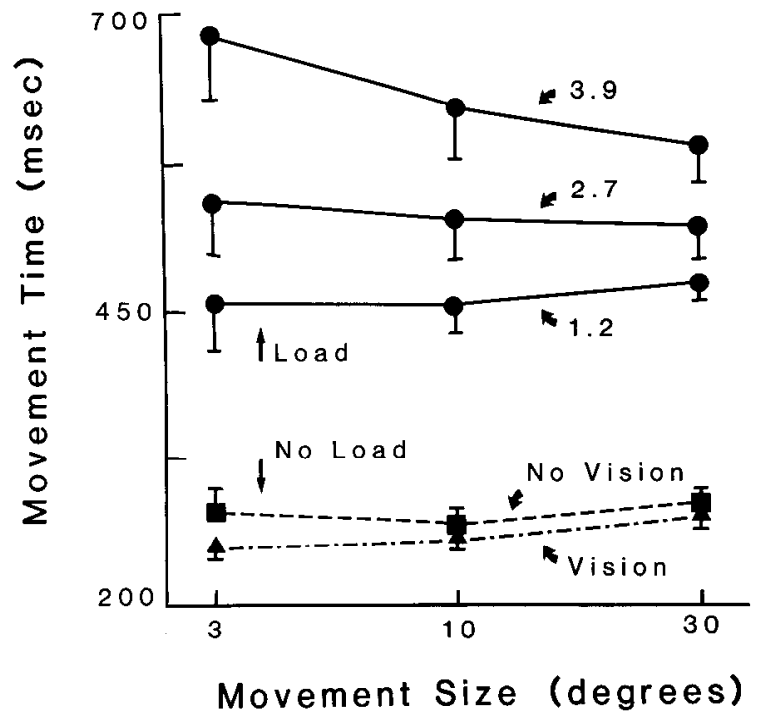

Figure 6. Movement time results. Numbers next to the arrows signify viscous load magnitude in units of $10^{-4} \mathrm{Nm} \mathrm{sec} \mathrm{deg}^{-1}$. Data not shown for the experiment in which permutation of presence or absence of visual guidance and load were the trial types. sulted in incomplete deafferentation (in those experiments muscle spindle function remained viable), thereby raising questions about their conclusions concerning the relative unimportance of somesthetic afferents for compensation to limb perturbations.

An implication of the results of Day and Marsden (1982) and
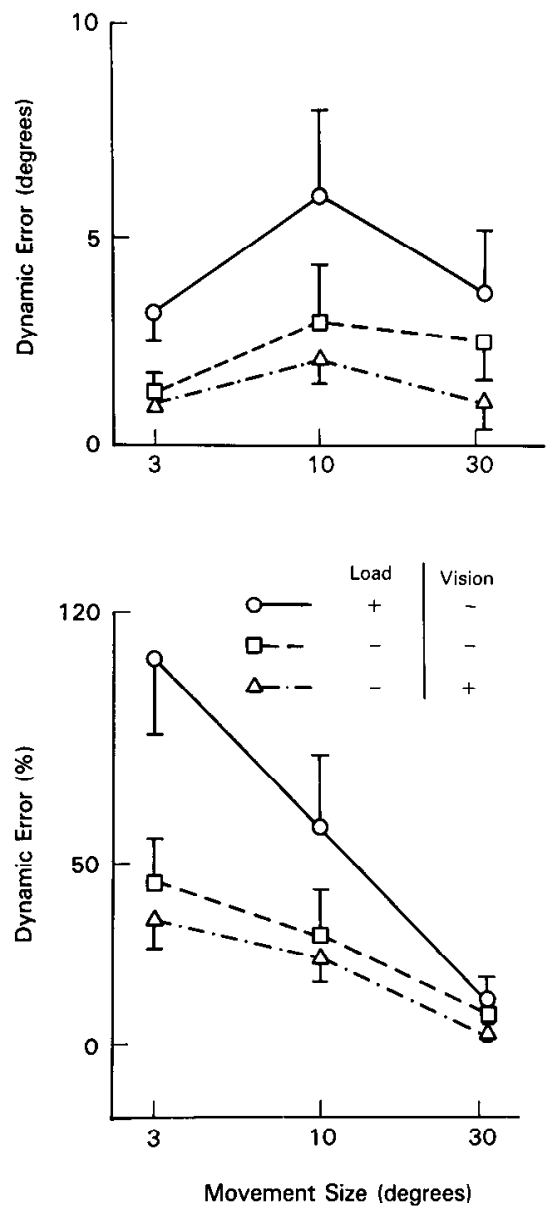

Figure 7. Dynamic error in degrees (top) and in proportion to movement size (bottom). Data are shown only for the experiment in which the heavicst load unexpectedly opposed movement. Plus and minus signs as in Figure 2. 
Rothwell et al. (1982a) is that mechanical perturbations occurring during movement that generate muscle responses will significantly affect the accuracy of perturbed movements. Day and Marsden (1982) showed rather conclusively that the absence of compensatory muscle responses following the introduction of an unexpected viscous load was related to inaccurate responses. Inaccurate limb positioning by patients affected with a largefiber sensory neuropathy, also without compensatory muscle responses, has also been described (Rothwell et al., 1982a; Sanes et al., 1985). The movements performed in the above experiments, though not extremely rapid, were considerably faster than those in experiments where perturbations encountered during movement failed to alter movement accuracy (Kelso and IIolt, 1980; Polit and Bizzi, 1979). Inspection of the EMG displays of intact preparations in these studies showed an absence of the large, short-latency muscle responses that have been observed in the present study and by others (Day and Marsden, 1982; Rothwell et al., 1982a) when movements were loaded uncxpectedly at movement onset. In addition, when, in the present experiment, triggered EMG responses were small following changes in viscous loading, as, for example, when light loads occurred unexpectedly, movement accuracy was unaffected by load changes. It would appear that perturbations that ordinarily fail to trigger muscle responses are ineffective in disrupting movement accuracy when subjects, because of disease or experimental procedures, are unable to generate compensatory muscle responses.

A further supposition concerning the mechanism of compensation for limb perturbations might be that the motor neuron pool is especially sensitive to perturbations occurring at or soon after the onset of the voluntary EMG (Gottlieb and Agarwal, 1980). Thus, peripheral inputs that influence the motor neuron pool would be more likely to affect the outcome of voluntary movement if they occurred synchronously with the motor command. Excitatory inputs from peripheral sources that arrive at the motor neurons after the descending input has decreased would have to contend with the diminished excitability of the motor neuron pool (for example, because of refractoriness, afterhyperpolarization, and active inhibition of motor neurons) and the absence of synergistic excitatory input, that is, the motor command. Thus, the failure of Kelso and Holt (1980) to observe significant effects of perturbations on movement in the intact limb could also have been related to the failure to introduce the perturbation early enough in the movement.

The above discussion is not intended to imply that the presence of involuntarily triggered muscle responses after limb perturbations should result in erroneous performance. Indeed, the EMG shown in Figure 5 indicated that the largest triggered muscle response (occurring when $30^{\circ}$ movements were loaded) had little effect on end-point positioning, while the relatively small muscle responses triggered during the performance of small movements had large effects on accuracy. It would appear, as suggested previously (Sanes and Evarts, 1983a, b), that the relationship between the voluntary and triggered EMG is crucial in determining whether an accurate end-point position will be achieved. Pursuing this line of reasoning, one would first reduce the neural circuit that controls motor performance to a simple (but perhaps, unrealistic) scheme, with 1 descending input, 1 peripheral input, and a relatively undifferentiated motor neuron pool. Small movements are caused by activation of a relatively small proportion of the motor neuron pool, whereas large movements are caused by activation of a relatively large portion of the pool. Thus, small movements probably recruit only lowthreshold motor units, while large movements recruit motor units with low and moderate thresholds. An additional observation on the organization of the motor neuron pool suggests why small movements are more affected by perturbation: The motor units in any pool with relatively low thresholds are more easily recruited and probably have more easily modulated discharge rates than high-threshold motor units. (Burke, 1981; Freund, 1983; Henneman and Mendell, 1981). If only lowthreshold motor units were recruited for small movements, excitatory peripheral inputs would be more likely to recruit motor units with slightly higher thresholds. In contrast, peripheral inputs occurring during large movements would have to raise the excitability level of the motor neuron pool to a significantly higher level than if small movements were performed to recruit new motor units. Thus, the summation of the motor command and the triggered EMG reaction would appear to be the important variable in determining whether movements were accurate.

The result, that proportionate end-point error increases with decreases in movement size while actual end-point error is relatively unvarying at about $2^{\circ}-5^{\circ}$, raises questions about whether error should be expressed proportionately or in degrees and about the relationship of end-point error to the resolution of position sense. We have argued previously that error should be expressed as a function of the movement size (Sanes and Evarts, 1983a). Since motor commands are scaled according to the demands of the task (Marsden et al., 1983), it would be expected that error would also be scaled in proportion to the intended movement (Craik, 1947). The finding that the proportionate end-point error of patients with a pathological large-fiber sensory neuropathy increased with decreases in movement size (Sanes et al., 1984, 1985) further supports the idea that errors should be expressed in relation to movement size. Since, in the present experiments, it was noted that end-point error ranged from $2^{\circ}$ to $5^{\circ}$, it could be argued that subjects did not perceive that they had deviated from the intended end-point. The limits of position sense at a variety of upper limb joints is about $2^{\circ}-$ $3^{\circ}$ for the detection of passive rotation across a wide range of movement velocities (Hall and McClosky, 1983) and this is very close to the observed end-point error for all movement sizes (except $10^{\circ}$ ) evaluated in the present experiment. Thus, it is possible that the final position achieved by subjects was within the kinesthetic noise and therefore undetectable by them. If this explanation could account for the present results, then end-point error would be expected to be distributed randomly about the intended final position, with the average end-point error equal to zero. However, the observation that almost all smaller movements ended beyond the intended final position argues against the notion that end-point error was within a zone of kinesthetic noise.

There is some question as to whether the present results could be explained, not on the basis of peripheral inputs interacting with motor commands, but as a consequence of mechanical properties of the forearm. These issues have been explored previously (Sanes and Evarts, 1983a) and it was noted that mechanical factors could not entirely account for results of the type presented here. If one assumes that the limb is a linear springlike system, then a purely mechanical explanation for performance with viscous loads would predict no difference between movements performed with or without viscous loads. Another potential explanation is that varying thresholds for proprioceptive receptors, or the different couplings between efferent discharge and afferent sensitivity at the different starting points for the $3^{\circ}$, $10^{\circ}$, and $30^{\circ}$ movements (Binder and Stuart, 1980; Botterman and Eldred, 1982; Ferrell, 1985; Kato et al., 1985), mediated greater relative overshoot for the smaller movements. However, one might have expected that, since the muscle spindles related to wrist flexors were in a more excitable initial state for the $30^{\circ}$ movement (this movement having begun at a more extended position) than for $3^{\circ}$ movements, that, if a threshold mechanism was operating, the predicted overshoot for the larger movements would have been greater than overshoot for the smaller movements. There is very little data on the response properties of 
muscle spindles during active movements by humans. The results of Hulliger et al. $(1982,1985)$ demonstrate that, whereas muscle spindle primaries and secondaries provide information about movement occurrence and direction, they often fail to signal precise positional information. Similarly, joint afferents would not be expected to provide a good index of joint position (Ferrell, 1985). The role of Golgi tendon organs in mediating the effects of viscous loads is uncertain. These receptors are exquisitely sensitive to changes in torque (Binder and Osborn, 1985; Houk and Henneman, 1967) and undoubtedly have a role in maintaining static postures; however, their role in active movement is far from clear (Appenteng and Prochazka, 1984).

A possible parallel exists between the results of the present experiments and those of Hollingworth (1909). He described an "illusion" of movement such that, when movements were blocked mechanically - presumably for no more than a few seconds - and then continued, subjects invariably overshot the intended end-point. Indeed, there is a remarkable correspondence between the data presented here and those of Hollingworth in that, in both studies, the proportion of error for blocked trials was related inversely to movement size. Granit (1972) discussed Hollingworth's (1909) work, attempting to explain the movement illusion on a large increase in muscle spindle discharge, as the movement was transformed from isotonic into isometric, which was monitored and processed by central motor structures and then caused triggered EMG responses. The central basis for spindle-triggered EMG responses (e.g., Evarts, 1973; Wolpaw, 1980) and the role of muscle spindles in movement illusions of a position sense (Goodwin et al., 1972; McCloskey, 1978 ) is well established. However, the actual role of muscle spindles versus that of other receptors in the mediation of motor performance errors is not entirely clear. If one borrows notions about the contributions of various somesthetic receptors to position and effort senses, then it could be said that all peripheral receptors contribute to movement regulation, but that muscle receptors probably have the greatest impact on movement control (Gandevia et al., 1983; Matthews, 1982).

The disruptive effects of unexpected perturbations on movement accuracy were reduced if subjects had some knowledge of results. In the experiment in which visual guidance was continuously available for some of the viscous-loaded trials, the disruptive effect of loading nonvisually guided trials was reduced. One possible reason for this result is that subjects made subtle alterations in movement strategy when responding to loadchanges when visual guidance was removed, in accordance with experience that was obtained when unexpected load changes occurred with visual guidance. It was noted that the early muscle responses were similar when the viscous-loading conditions were changed regardless of the visual guidance condition. IIowever, the late responses, commencing at latencies greater than 100 msec, were different, suggesting that voluntary reactions are the most reliable means of compensating for unexpected perturbations occurring during movement (Marsden et al., 1978; Rothwell et al., 1982b).

\section{References}

Appenteng, E., and A. Prochazka (1984) Tendon organ firing during active muscle lengthening in awake, normally behaving cats. J. Physiol. (Lond.) 353: 81-92.

Binder, M. D., and C. E. Osborn (1985) Interactions between motor units and Golgi tendon organs in the tibialis posterior muscle of the cat. J. Physiol. (Lond.) 364: 199-215.

Binder, M. D., and D. G. Stuart (1980) Responses of Ia and spindle Group II afferents to single motor-unit contractions. J. Neurophysiol. 43: 621-629.

Botterman, B. R., and E. Eldred (1982) Static stretch sensitivity of Ia and II afferents in the cat's gastrocnemius. Pfluegers Arch. 395: 204211.

Burke, R. E. (1981) Motor units: Anatomy, physiology, and functional organization. In Handbook of Physiology: The Nervous System II, J. M. Brookhart, V. B. Mountcastle, and S. R. Geiger, eds., pp. 345422, Waverly Press, Baltimore, MD.

Craik, K. J. W. (1947) Theory of the human operator in control systems. I. The operator as an engineering system. Br. J. Psychol. 38: $56-61$.

Day, B. L., and C. D. Marsden (1982) Accurate repositioning of the human thumb against unpredictable dynamic loads is dependent upon peripheral feed-back. J. Physiol. (Lond.) 327: 393-407.

Evarts, E. V. (1973) Motor cortex reflexes associated with learned movements. Science 179: 501-503.

Ferrell, W. R. (1985) The response of slowly adapting mechanoreceptors in the cat knee joint to tetanic contraction of hind limb muscles. Q. J. Exp. Physiol. 70: 337-345.

Freund, H.-J. (1983) Motor unit and muscle activity in voluntary motor control. Physiol. Rev. 63: 387-436.

Gandevia, S. C., L. A. Hall, D. I. McCloskey, and E. K. Potter (1983) Proprioceptive sensation at the terminal joint of the middle finger. J. Physiol. (Lond.) 335: 507-517.

Goodwin, G. M., D. I. McCloskey, and P. B. C. Matthews (1972) The contribution of muscle afferents to kinesthesia shown by vibration induced illusions of movement and by effects of paralysing joint afferents. Brain 95: 705-748.

Gottlieb, G. L., and G. C. Agarwal (1970) Filtering of electromyographic signals. Am. J. Physiol. Med. 49: 142-146.

Gottlieb, G. L., and G. C. Agarwal (1980) Response to sudden torques about ankle in man. III. Suppression of stretch-evoked responses during phasic contraction. J. Neurophysiol. 44: 233-246.

Granit, R. (1972) Constant errors in the execution and appreciation of movement. Brain 95: 649-660.

Hall, L. A., and D. I. McCloskey (1983) Detections of movements imposed on finger, elbow and shoulder joints. J. Physiol. (Lond.) 335: 519-533.

Hasan, Z., and R. M. Enoka (1985) Isometric torque angle relationship and movement-related activity of human elbow flexors: Implications for the equilibrium-point hypothesis. Exp. Brain Res. 59: 441-450.

Hasan, Z., and J. C. Houk (1975) The transition in the sensitivity of spindle receptors that occurs when a muscle is stretched more than a fraction of a millimeter. J. Neurophysiol. 38: 673-689.

Henneman, E., and L. M. Mendell (1981) Functional organization of motoneuron pool and its inputs. In Handbook of Physiology: The Nervous System II, J. M. Brookhart, V. B. Mountcastle, and S. R. Geiger, eds., pp. 423-503, Waverly Press, Baltimore, MD.

Hollingworth, E. L. (1909) The inaccuracy of movement. Arch. Psychol. (13), 1-87.

IIouk, J., and E. IIenneman (1967) Responses of Golgi tendon organs to active contractions of the soleus muscle of the cat. J. Neurophysiol. 30: $466-481$

Hulliger, M., E. Nordh, and $\AA$. B. Vallbo (1982) The absence of position response in spindle afferent units from human finger muscles during accurate position holding. J. Physiol. (Lond.) 322: 167-179.

Hulliger, M., E. Nordh, and $\AA$. B. Vallbo (1985) Discharge of muscle spindle afferents related to direction of slow precision movements in man. J. Physiol. (Lond.) 362: 437-453.

Kato, M., S. Murakami, and K. Yasuda (1985) Behavior of single motor units of human tibialis anterior muscle during voluntary shortening contraction under constant load torque. Exp. Neurol. 90: 238253.

Kelso, J. A. S., and K. G. Holt (1980) Exploring a vibratory system analysis of human movement production. J. Neurophysiol. 43: 11831196.

Knibestöl, M., and $\AA$. B. Vallbo (1980) Intensity of sensation related to activity of slowly adapting mechanoreceptive units in the human hand. J. Physiol. (Lond.) 300: 251-267.

Laszlo, J. I., and P. J. Bairstow (1971) Accuracy of movement, peripheral feedback and efference copy. J. Motor Behav. 3: 241-252.

Lestienne, F., A. Polit, and E. Bizzi (1981) Functional organization of the motor process underlying the transition from movement to posture. Brain Res. 230: 121-131.

Marsden, C. D., P. A. Merton, H. B. Morton, J. E. R. Adam, and M. Hallett (1978) Automatic and voluntary responses to muscle stretch in man. Prog. Clin. Neurophysiol. 4: 167-177.

Marsden, C. D., J. A. Obeso, and J. C. Rothwell (1983) The function of the antagonist muscle during fast limb movements in man. J. Physiol. (Lond.) 335: 1-13.

Matheson, J., M. Hallett, A. Berardelli, R. Weinhaus, and S. Inzucchi 
(1985) Failure to confirm a correlation between electromyogram and final position. Hum. Neurobiol. 4: 257-260.

Matthews, P. B. C. (1982) Where does Sherrington's "muscular sense" originate? Muscles, joints, corollary discharges? Annu. Rev. Neurosci. 5: $189-218$.

Matthews, P. B. C., and R. B. Stein (1969) The sensitivity of muscle spindle afferents to small sinusoidal changes of length. J. Physiol. (Lond.) 200: 723-743.

McCloskey, D. I. (1978) Kinesthetic sensibility. Physiol. Rev. 58:763820 .

Nam, M. H., V. Lakshminarayanan, and L. W. Stark (1984) Effect of external viscous load on head movement. IEEE Trans. Biomed. Eng. 31: 303-309.

Polit, A., and E. Bizzi (1979) Characteristics of motor programs underlying arm movements in monkeys. J. Neurophysiol. 42: 183-194.

Rothwell, J. C., M. M. Traub, B. L. Day, J. A. Obeso, P. K. Thomas, and C. D. Marsden (1982a) Manual motor performance in a deafferented man. Brain 105: 515-542.

Rothwell, J. C., M. M. Traub, and C. D. Marsden (1982b) Automatic and "voluntary" responses compensating for disturhances of human thumb movments. Brain Res. 248: 33-41.

Sanes, J. N. (1983) Inaccurate limb positioning after unexpected perturbations during movement. Soc. Neurosci. Abstr. 9: 630 .

Sanes, J. N., and E. V. Evarts (1983a) Effects of perturbations on accuracy of arm movements. J. Neurosci. 3: 977-986.

Sanes, J. N., and E. V. Evarts (1983b) Regulatory role of proprioceptive input in motor control of phasic or maintained voluntary contractions in man. In Motor Control Mechanisms in Health and Disease, J. E. Desmedt, ed., pp. 47-59, Kaven, New York.

Sanes, J. N., K.-H. Mauritz, E. V. Evarts, M. C. Dalakas, and A. Chu (1984) Motor deficits in patients with large-fiber sensory neuropathy. Proc. Natl. Acad. Sci. USA 81: 979-982.

Sanes, J. N., K.-H. Mauritz, M. C. Dalakas, and E. V. Evarts (1985) Motor control in humans with large-fiber sensory neuropathy. Hum. Neurobiol. 4: 101-114.

Wolpaw, J. R. (1980) Correlation between task-related activity and responses to perturbations in primate sensorimotor cortex. J. Neurophysiol. 44: 1122-1138. 